\title{
Positive Effects of
} Mindfulness-Based Training on Energy Maintenance and the EEG Correlates of Sustained Attention in a Cohort of Nurses

OPEN ACCESS

Edited by:

Nandini Chatterjee Singh, National Brain Research Centre (NBRC), India

Reviewed by: Herman Nico Logemann, Eötvös Loránd University, Hungary Dan Zhang, Tsinghua University, China

*Correspondence: Julian Lim julian.lim@duke-nus.edu.sg

Received: 20 June 2017 Accepted: 14 February 2018 Published: 01 March 2018

Citation:

Wong KF, Teng J, Chee MWL, Doshi K and Lim J (2018) Positive Effects of Mindfulness-Based Training on Energy Maintenance and the EEG Correlates of Sustained Attention in a Cohort of Nurses.

Front. Hum. Neurosci. 12:80. doi: 10.3389/fnhum.2018.00080

\author{
Kian F. Wong ${ }^{1}$, James Teng ${ }^{1}$, Michael W. L. Chee ${ }^{1}$, Kinjal Doshi ${ }^{2}$ and Julian Lim ${ }^{1 *}$ \\ ${ }^{1}$ Center for Cognitive Neuroscience, Neurosciences and Behavioral Disorders Department, Duke-NUS Medical School, \\ Singapore, Singapore, ${ }^{2}$ Department of Neurology, Singapore General Hospital, Singapore, Singapore
}

Mindfulness based training (MBT) is becoming increasingly popular as a means to improve general wellbeing through developing enhanced control over metacognitive processes. In this preliminary study, we tested a cohort of 36 nurses (mean age $=30.3$, $\mathrm{SD}=8.52$; 2 male) who participated in an 8-week MBT intervention to examine the improvements in sustained attention and its energetic costs that may result from MBT. Changes in sustained attention were measured using the psychomotor vigilance task (PVT) and electroencephalography (EEG) was collected both during PVT performance, and during a brief period of meditation. As there was substantial variability in training attendance, this variable was used a covariate in all analyses. Following the MBT program, we observed changes in alpha power across all scalp regions during meditation that were correlated with attendance. Similarly, PVT performance worsened over the 8-week period, but that this decline was mitigated by good attendance on the MBT program. The subjective energy depletion due to PVT performance (measured using self-report on Likert-type scales) was also less in regular attendees. Finally, changes in known EEG markers of attention during PVT performance (P300 and alpha-band event-related desynchronization) paralleled these behavioral shifts. Taken together, our data suggest that sustained attention and its associated costs may be negatively affected over time in the nursing profession, but that regular attendance of MBT may help to attenuate these effects. However, as this study contained no control condition, we cannot rule out that other factors (e.g., motivation, placebo effects) may also account for our findings.

\footnotetext{
Keywords: mindfulness-based training, sustained attention, psychomotor vigilance test, P300, event-related desynchronization, nurses
}

\section{INTRODUCTION}

Sustained attention describes the ability to focus on task-relevant behavior and resist distractibility, particularly over long periods of time. Despite the importance of sustaining attention in many on-the-job situations, attentional failures in such settings are common due to factors such as mind-wandering, motivational decline and fatigue, which can in turn lead to serious accidents 
(Dinges, 1995). The medical profession is a textbook example of this: impairments in sustained attention in medical settings can result in errors that may seriously compromise the safety and well being of patients. For instance, a review of the different errors made by nurses found that lack of attentiveness was an important contributor to mistakes such as missing predictable complications and inadequate monitoring of patients, with potentially catastrophic results (Benner et al., 2002). Given its importance, we conducted a study to assess whether sustained attention in nurses might be improved via mindfulness training, an intervention that may have associated cognitive benefits (Chiesa et al., 2011).

\section{Mindfulness Training and Sustained Attention}

Traditional countermeasures to prevent dangerous lapses in attention include taking rest breaks (Tucker et al., 2003) or using stimulants such as caffeine (Bonnet et al., 1995). However, both of these are imperfect and short-term solutions. Mindfulness-based training (MBT), which focuses on developing non-judging meta-awareness of present-moment experience, (Kabat-Zinn et al., 1985) represents a promising alternative for mitigating declines in sustained attention (Chiesa and Serretti, 2010). MBT seems especially appealing as a means of stabilizing attention, as one of the skills explicitly taught in this program is how to return attention to a particular locus following the observation that the mind has wandered. Furthermore, heightened mindfulness cultivates higher levels of meta-awareness over time (Jankowski and Holas, 2014), which should in turn also lead to enhancements in sustained attention.

Despite these intuitions, studies of the effects of mindfulness on sustained attention have revealed inconsistent results, with some positive (Valentine and Sweet, 1999; Josefsson and Broberg, 2011; Tha et al., 2015) and some null findings (Tang et al., 2007; MacCoon et al., 2014). These conflicting results motivated us to conduct the current experiment using the psychomotor vigilance test (PVT; Dinges and Powell, 1985), which has unique advantages as an assay of sustained attention. In particular, successful performance of this test does not heavily require other facets of attention (e.g., orienting and/or executive attention) as compared with paradigms such as the continuous performance task (CPT; MacCoon et al., 2014) or the Sustained Attention to Response Task (SART; Jha et al., 2015). Furthermore, an earlier study showed that PVT performance improved in novice meditators following $40 \mathrm{~min}$ of meditation, compared to $40 \mathrm{~min}$ of nap or eyes opened rest (Kaul et al., 2010). Finally, the PVT is highly valid in predicting real-world performance and is already used to assess the level of impairment faced by individuals under conditions of fatigue (Lim and Dinges, 2008) and during important operations (Dinges, 2016).

The most sensitive summary metrics of PVT performance are response speed (reciprocal reaction time, or 1/RT), and the number of lapses, or reaction times over $500 \mathrm{~ms}$ (Basner and Dinges, 2011). Additionally, reaction times typically increase over the course of a PVT bout, a phenomenon known as the time-on-task effect (Parasuraman and Jiang, 2012). This effect is attributed to the withdrawal of attention, due in some measure to resource limits, but also to effort reallocation (Thomson et al., 2015).

In addition to measuring PVT performance, we also considered the subjective effort expended by participants using Likert-type scales assessing energy and mood. Paying attention to a task for a prolonged period of time is effortful and has a subjective energetic cost (Warm et al., 2008), exacerbating the difficulty in situations where such a lengthy period of stable attention is needed.

\section{Electrophysiological Correlates of Attention and Meditation}

Changes in sustained attention are detectable both in behavioral and electroencephalographic (EEG) data collected during PVT performance. Objective neurophysiological markers of change are favored in mindfulness research, as they are generally less susceptible to experimenter effects and other biases. In the current study, we used two established EEG markers of attention to assess the endogenous effects of mindfulness training. First, the P3 event related potential (ERP) is often associated with directed attention (Polich and Kok, 1995), and declines in P3 amplitude reflect a reduction in the ability to allocate attention to on-task behavior due to time-on-task (Möckel et al., 2015). In vigilance paradigms, P3 amplitude is significantly greater for detected targets than omission errors (Davies and Parasuraman, 1977). Second, alpha band activity is suggested to reflect background neural noise which blocks cognitive processing (Klimesch, 1999), and individuals who display reduce alpha synchronization following a critical event show better processing of that stimulus (Yordanova et al., 2001).

Both alpha and theta power during meditation increase following training, with theta band power changes observed in individuals highly experienced in meditation (Lomas et al., 2015). In the present study, the effectiveness of 8 weeks of meditation training should be reflected in the change in electrophysiological power during meditation.

\section{Cognitive Benefits of Mindfulness for Nurses}

Attentiveness is the key to good nursing practice (Benner et al., 2002) as nurses have to monitor therapies and patient responses over long periods and across multiple patients. Failures in monitoring may lead to both errors of omission and commission in medical practice. This becomes more prominent when nurses become tired or distracted, which are two of the three most reported causes of medical errors (Mayo and Duncan, 2004). Furthermore, lapses of attention are the most common reason for mistakes in drug administration (Härkänen et al., 2013). We believe that MBT training for nurses could lead to improvements in sustained attention, which may subsequently translate into improved patient outcomes. At the same time, we note that MBT can be challenging for some participants to adhere to, and compliance with the program may be particularly difficult in workplace settings (Van Dongen et al., 2016).

Building on the observations above, the present study examines the effects of an 8-week MBT course on PVT 
performance, EEG activity, and subjective energy maintenance in a group of nurses from a local public hospital. We hypothesized that PVT reaction times would improve following the intervention, that these changes would also be reflected in P3 amplitude to PVT targets, and that subjective energy expenditure on the task would decrease. Additionally, we hypothesized that these changes would be moderated by the amount of meditation training received.

\section{MATERIALS AND METHODS}

Forty-six nurses from a local government hospital were recruited to participate in this study. Of these, 10 were excluded from the analysis as they did not return for the post-intervention data collection. The remaining 36 nurses (mean age $=30.3$, $\mathrm{SD}=8.52 ; 2$ male) provided behavioral and EEG data in both experimental sessions, which occurred before (S1) and after (S2) a MBT program (Figure 1A). The nurses had been employed in the profession for an average of 8.52 years, with a skew towards junior nurses: 24 of the participants had been in their position for fewer than 5 years. Data were collected at the same time of day at each session for each participant to control for circadian effects. All of the nurses were shift workers, but testing sessions never occurred directly after completion of a shift. Data collection took about $2 \mathrm{~h}$ to complete per session and participants were compensated $\$ 40$ for their time for each session. The study was approved by the Singhealth Centralized Institutional Review Board and was conducted in accordance with the ethical standards of the 1964 Helsinki declaration and its later amendments. All participants provided written informed consent.

\section{Mindfulness Intervention}

Eight weekly 90-min sessions of MBT were conducted by an experienced mindfulness instructor (Figure 1B). These sessions were based on the mindfulness-based stress reduction (MBSR) program developed by Kabat-Zinn et al. (1985) and consisted of instruction in mindfulness practices, group sharing, and in-class activities. Both formal (e.g., mindful breathing, body scan) and informal (communication with compassion) exercises were taught as part of this program. Participants were strongly encouraged to practice the techniques taught in class for at least $15 \mathrm{~min}$ a day at home.

The key differences between the intervention used here and manualized MBSR were: (1) shorter session duration (1.5 vs. $2.5 \mathrm{~h}$ ); (2) shorter duration of practice (15-20 $\mathrm{min} /$ day vs. $45 \mathrm{~min}$ per day); and (3) our intervention did not contain a retreat.

\section{Behavioral and EEG Data Collection}

Sixty-four-channel EEG data using the standard 10-20 electrode positioning system were collected using a BrainProducts MR+ amplifier with an actiCAP at $250 \mathrm{~Hz}$ sampling rate, referenced at $\mathrm{FCz}$ and ground at Fpz. All electrode impedances were brought below $10 \mathrm{k} \Omega$ before the start of the recording. Five blocks of data were collected (Figure 1C): 4 min of eyes-open task-free activity, $4 \mathrm{~min}$ of eyes-closed task-free activity, $4 \mathrm{~min}$ of eyes-closed meditation with auditory instruction, a 20-min PVT (Figure 1D; Dinges and Powell, 1985; Dinges and Kribbs, 1991), and a second 4 min of eyes-open task-free activity. During the task-free (resting state) scans, participants were asked to maintain their gaze on a fixation cross on the computer monitor, and not to think of anything in particular. Subjective behavioral scales measuring energy and mood (Lim et al., 2012) were
A

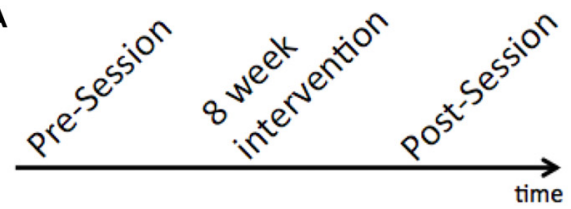

B 8 week intervention

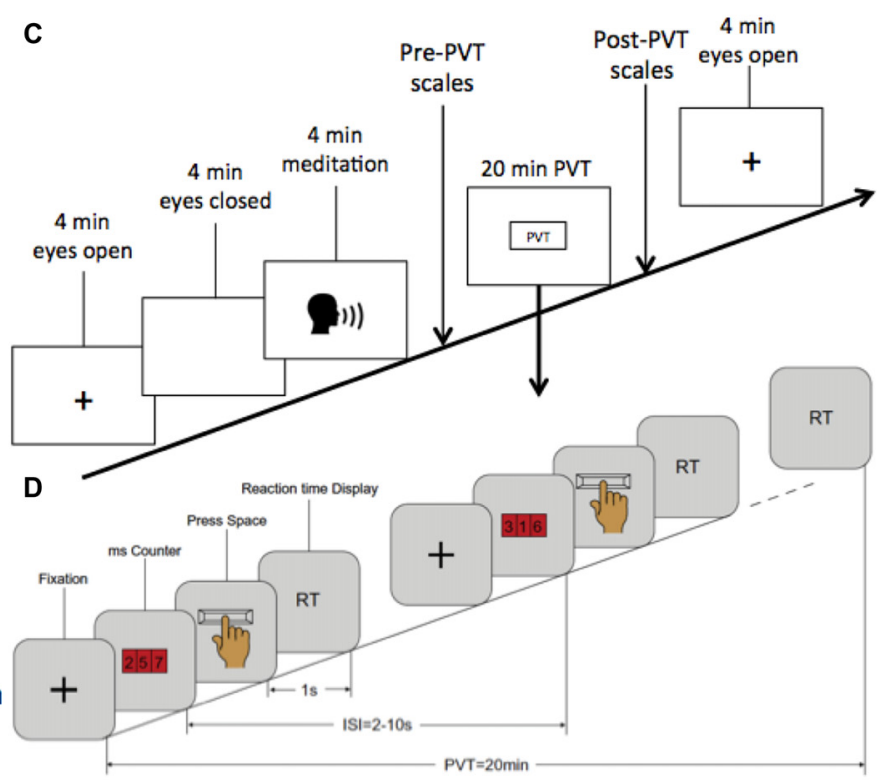

FIGURE 1 | Schematics of data collection and the mindfulness-based training (MBT) program. (A) Sequence of sessions attended by participants. (B) The theme of each of the intervention sessions. (C) Sequence of blocks of electroencephalography (EEG) data collection across sessions. (D) Schematic of the psychomotor vigilance task $(\mathrm{PVT})$ task. 
administered before and after the PVT. Additionally, sleep and wake times for the past 2 days before each recording session were collected.

\section{Psychomotor Vigilance Task}

The PVT is a demanding test of sustained attention that is predictive of attentional lapses in operational settings (Dorrian et al., 2005; Lim and Dinges, 2010). During the PVT, participants monitor a fixed point on a display for the appearance of a millisecond counter. They are instructed to respond as quickly as possible to this counter without anticipating its appearance, following which their response time remains on screen for $1000 \mathrm{~ms}$. Participants responded using the spacebar on a standard QWERTY keyboard with their dominant hand. A 20-min version of the PVT was administered to increase between-subject variance in fatigue and time-on-task (Lim et al., 2010). All stimuli were programed and presented using Psychtoolbox (Brainard, 1997).

\section{Behavioral Analysis}

Due to the wide range of MBT sessions attended (0-8), this variable was used as a covariate in all subsequent analyses. Median reaction speed (RS), and lapses (responses >500 ms) were calculated for each participant and values for each session were entered into a one-way repeated measures ANCOVA. Subjective energy and mood scores were entered into a 2-way repeated measures ANCOVA with time (pre and post PVT) and session as factors. We correlated attendance (number of sessions) with the change in performance (response speed and lapse count) from pre- to post-intervention. We also performed correlations of attendance with the subjective measures of energy and mood.
TABLE 1 | Electrodes in each region.

\begin{tabular}{ll}
\hline Region/cluster & Electrodes \\
\hline Frontal & Fp1 Fp2 AF3 AF4 Fz \\
Central & FC1 FC2 C3 C4 Cz \\
Parietal & CP1 CP2 P3 P4 Pz \\
Left temporal & F3 F7 FC5 T7 CP5 P7 \\
Right temporal & F4 F8 FC6 T8 CP6 P8 \\
Occipital & PO3 PO4 O1 O2 Oz \\
\hline
\end{tabular}

Reported sleep times were rounded to the closest quarter hour and average sleep duration for the two nights before the EEG session was calculated. A paired sampled $t$-test was used to compare sleep duration before the pre-intervention session and before the post-intervention session.

\section{EEG Data Processing and Analysis}

EEG data were processed in EEGLAB (Delorme and Makeig, 2004). Data were re-referenced offline to the average of mastoids $((\mathrm{A} 1+\mathrm{A} 2) / 2)$ and band pass filtered at 1-30 Hz. Infomax ICA was used to identify and remove eye movement components from the data. The individual alpha frequency (IAF; Klimesch, 1999) of each participant was identified and the individual alpha band was defined as the bandwidth $2 \mathrm{~Hz}$ above and $2 \mathrm{~Hz}$ below the IAF. Individual theta band was defined as the range from $4 \mathrm{~Hz}$ to $6 \mathrm{~Hz}$ below the IAF (Klimesch, 1999).

To assess whether MBT had an effect on brain activity when participants entered a mindful state, the power spectrum of the EEG data during meditation was calculated using the EEGLAB "spectopo" function (512 point FFT with 50\% overlap) across six scalp regions (Table 1). This function uses Welch's method to estimate spectral density (Welch, 1967). The change of the mean power in the individual alpha band (Figure 2;

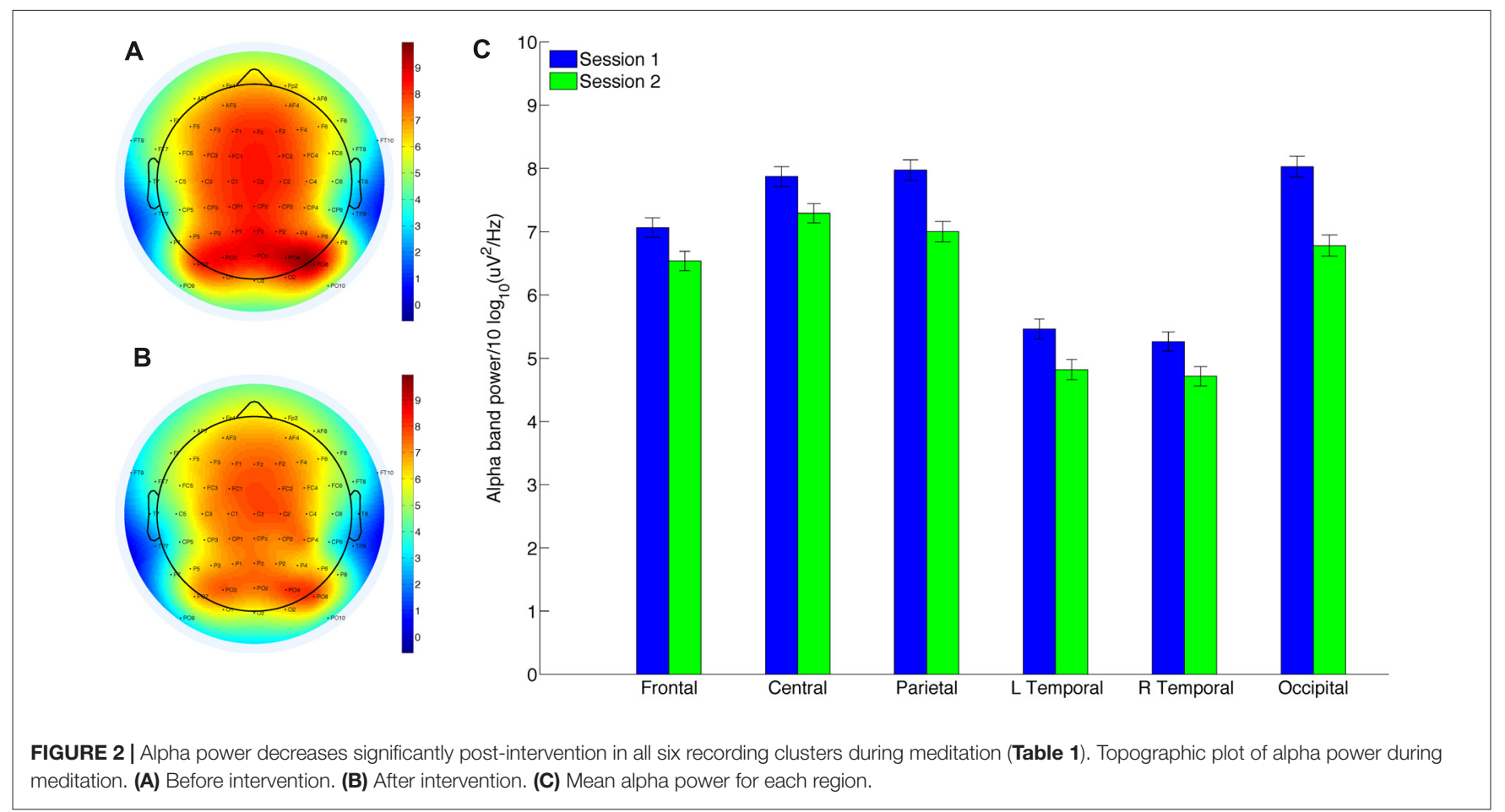


TABLE 2 | Results comparing individual alpha power during 4 min of guided meditation before and after mindfulness-based training (MBT) with FDR adjusted Q values.

\begin{tabular}{lllllll}
\hline & Frontal & Central & Parietal & Left temporal & Right temporal & Occipital \\
\hline$F_{(1,34)}(\mathrm{Q}$ value) & $14.716(0.0008)^{* *}$ & $17.224(0.0001)^{* *}$ & $18.620(0.00002)^{* *}$ & $13.988(0.001)^{* *}$ & $17.176(0.0001)^{* *}$ & $17.977(0.00005)^{* *}$ \\
Correlation with attendance (Q value) & $0.494(0.001)^{* *}$ & $0.519(0.0002)^{* *}$ & $0.456(0.003)^{*}$ & $0.462(0.004)^{* *}$ & $0.524(0.0003)^{* *}$ & $0.436(0.008)^{* *}$ \\
Effect Size $\eta^{2}$ & 0.302 & 0.336 & 0.354 & 0.291 & 0.336 & 0.346
\end{tabular}

** Significant at 0.01 level; * significant at 0.05 level.

Table 2) and the individual theta band before and after intervention at each scalp region was tested using repeated measures ANCOVA.

P3 (mean amplitude over 300-400 ms) ERPs were calculated using a $-200 \mathrm{~ms}$ to stimuli onset baseline at channel $\mathrm{Pz}$. Epochs were automatically flagged for removal if amplitude exceeded 3 standard deviations from the mean; visual inspection for noise was a secondary measure used prior to removal of these trials. Thirty-four of 4322 trials $(0.79 \%)$ were rejected. Event related desynchronization (ERD; Pfurtscheller and Lopes da Silva, 1999), defined as mean percentage synchronization change 200-500 ms post trial onset using individual alpha bands was calculated at Pz. Mean ERP and ERD amplitude were computed for the first and last $4 \mathrm{~min}$ of the PVT to assess the effect of time-on-task (referred to in the ANCOVAs as "time"); we analyzed the data this way to minimize the confounding effects of between-session shifts in mean ERP/ER amplitude. Both ERP and ERD data were entered into a $2 \times 2$ time-by-session ANCOVA. ERP and ERD changes over time across sessions were also correlated to the number of sessions attended.

All dependent variables were evaluated for normality using Shapiro-Wilk: P3 ERP amplitudes were not normally distributed and a non-parametric (Spearman) test was used when correlating this variable.

\section{Subjective Scales}

Subjective sensations were measured using 9-point Likert-type scales asking for ratings of sleepiness, fatigue, energy, motivation, depression, anxiety and stress; factor analysis has previously shown these to load onto two subscales: energy and mood (Lim et al., 2012). Measures from before and after PVT performance in S1 and S2 were collected and entered into a $2 \times 2$ repeatedmeasures ANCOVA.

\section{RESULTS}

\section{Training Attendance}

Although the importance of regular attendance was stressed to study participants, the number of sessions attended overall was relatively low (mean $(\mathrm{SD})=4.14(2.33)$; range $=0-8)$. The most common reason for missing a training session was conflict with a work shift, as these tend to be unpredictable in the population under study. To account for the large variance in sessions attended, we included this variable as a covariate in the analysis of our behavioral and EEG data, and report statistics for one-way repeated measures ANCOVA rather than main effects.

\section{Sleep Duration}

Self reported sleep durations were collected for the two nights prior to each EEG testing session. We found no differences between the averaged sleep duration per night prior to the two recording sessions (S1 (SD): $431.5 \mathrm{~min}$ (71.2); S2: $440.6 \mathrm{~min}$ (78.0), $t=-0.538, p=0.594, d=-0.0922)$.

\section{EEG Spectral Analysis of Meditation}

We assessed whether the EEG power spectrum of participants differed when they were instructed to enter a state of mindfulness after the 8-week period of training. To test this, we compared both alpha and theta power over six scalp regions during meditation in S1 and S2. Alpha power changed significantly across all regions (Figure 2) after correction for multiple comparisons and the degree of change was significantly correlated with MBT attendance over all regions (Table 2). Although there was a group decrease in alpha power across sessions, examination of individual differences showed that participants with higher attendance tended to have relatively stable or increased levels of alpha in S2 (Table 2). Theta power changes from S1 to S2 were not significant across any region, and were not correlated with MBT attendance.

\section{Psychomotor Vigilance Test Performance}

To assess performance on the PVT, we extracted two variables from each 20-min test bout: median response speed (RS; reciprocal $\mathrm{RT}$, or $1 / \mathrm{RT}$, with $\mathrm{RT}$ measured in milliseconds), and lapses. These variables are the most sensitive markers of sustained attention in this task (Basner and Dinges, 2011), and are also highly reliable over time (Dorrian et al., 2005).

Compared with previous studies of participants in a well-rested state (Lim and Dinges, 2010), we observed that average performance on the PVT was relatively poor (median (standard deviation (SD)) RS: 2.917 (0.280) $\mathrm{ms}^{-1}$; lapses: 12.85 (12.73)). While we would traditionally reject participants who commit at least 20 or more lapses (RT > 500 ms; Lim et al., 2012), here, this would result in the removal of 13 participants from the sample of 36 .

Inspection of the data suggested that the high lapse count in this dataset was driven by generalized response slowing, and not stochastic attentional failures. As such, we used a more liberal threshold for classifying lapses, which was defined as the median +2 SD of the RT (mean (SD) RT $=711(62) \mathrm{ms}$ ) of the PVT performance in S1, calculated individually for each participant. This individual lapse threshold was applied to both S1 (mean (SD) lapse count $=7.6(3.5)$ ) and S2 (mean (SD) lapse count $=9.81(8.75)$ ) PVT results. Using this criterion, only one remaining participant committed an excessive number of 

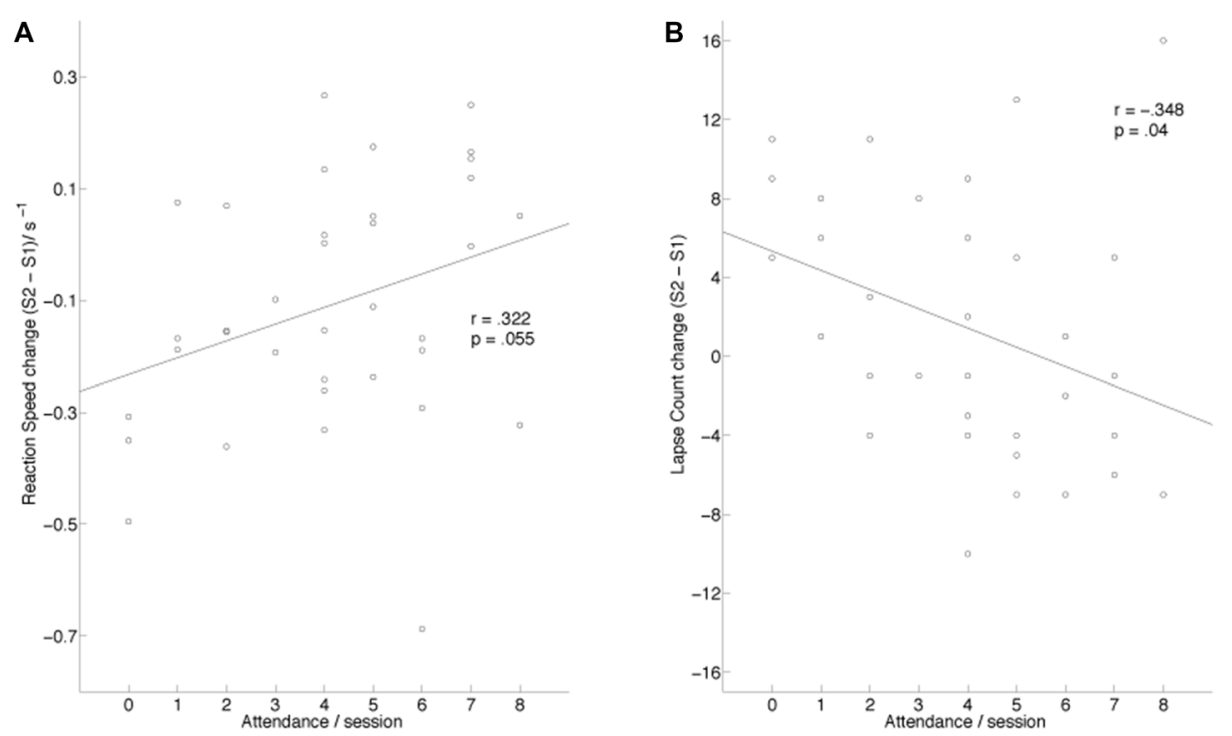

FIGURE 3 | (A) Change in median response speed (S2-S1) is significantly correlated with MBT attendance. (B) Change in number of lapses (S2-S1) is significantly correlated with MBT attendance.
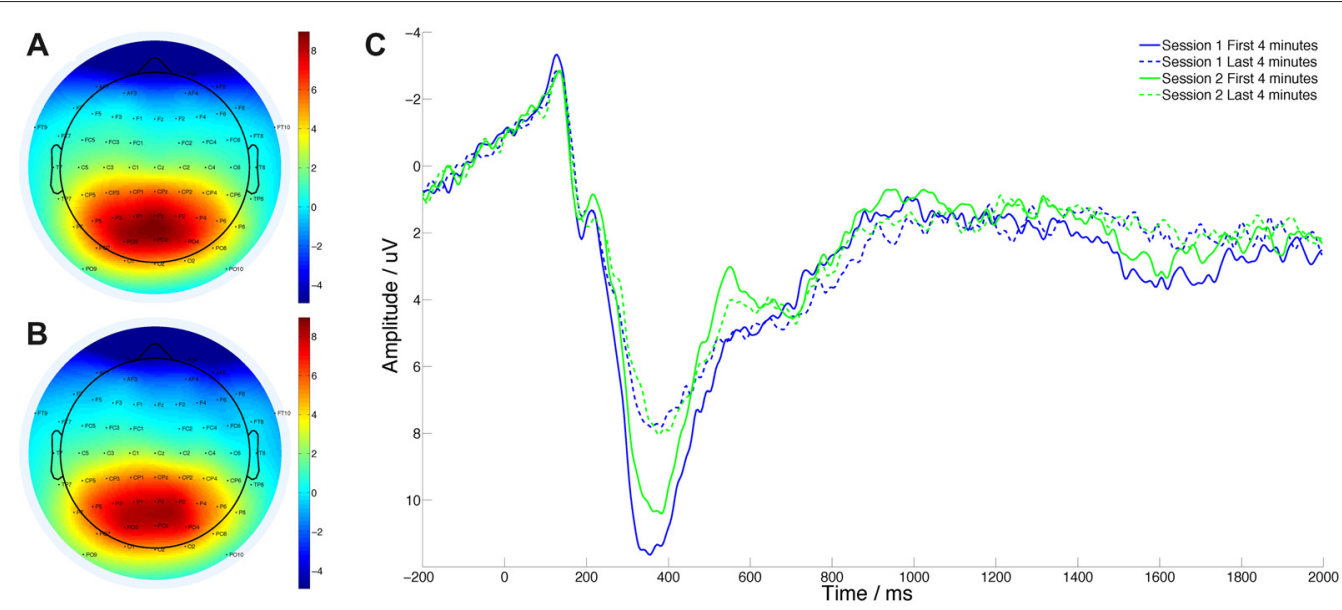

FIGURE 4 | Grand average P3 EEG amplitude topographic plot during PVT. (A) Before intervention. (B) After intervention. (C) Grand average event related potential (ERP) at channel Pz.

lapses in S2 PVT (40), 3.5 SD. over the mean; this dataset was removed from further analysis that required lapse trials to be rejected.

Using one-way ANCOVA, we found a significant effect of session on RS $\left(F_{(1,34)}=10.571, p=0.003, \eta_{\mathrm{p}}^{2}=0.237\right)$ and lapses $\left(F_{(1,33)}=6.177, p=0.018, \eta_{\mathrm{p}}^{2}=0.158\right)$. On the average, performance in S2 was worse, with slower RS (S1 median (SD) RS: 2.97 (0.29); S2 median (SD) RS: 2.86 $(0.28)$ ) and more lapses (S1 mean (SD): 7.60 (3.45); S2 mean (SD): 8.94 (7.16)). To assess the direction of effect of the covariate, we correlated attendance with change in response speed (Figure 3A) and lapses (Figure 3B) and found a marginally significant correlation with RS $(r=0.322, p=0.055)$ and a significant correlation with lapses $(r=-0.348, p=0.04)$. Higher attendance was associated with better performance in S2 vs. S1 for both RS and lapse counts. We note from the scatter plots that this correlation was driven both by improved performance in regular attendees as well as degraded performance in individuals who attended little to none of the program.

\section{ERP Analysis}

P3 amplitude to correct, non-lapse responses was measured and averaged for the first and last $4 \mathrm{~min}$ of each 20-min 
A

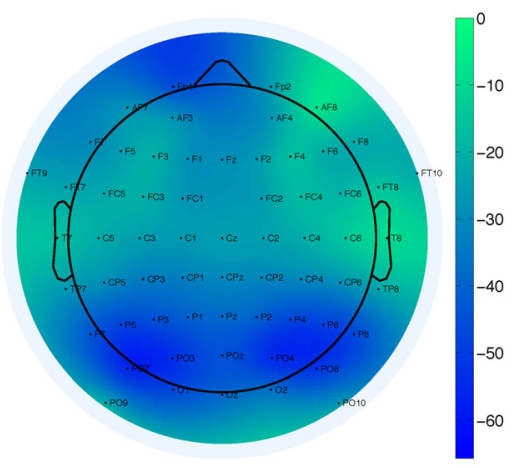

B

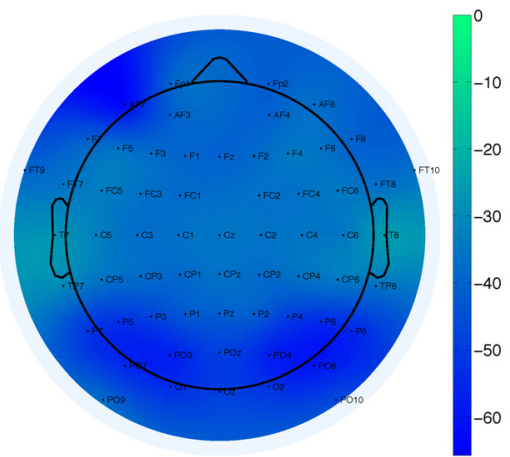

C

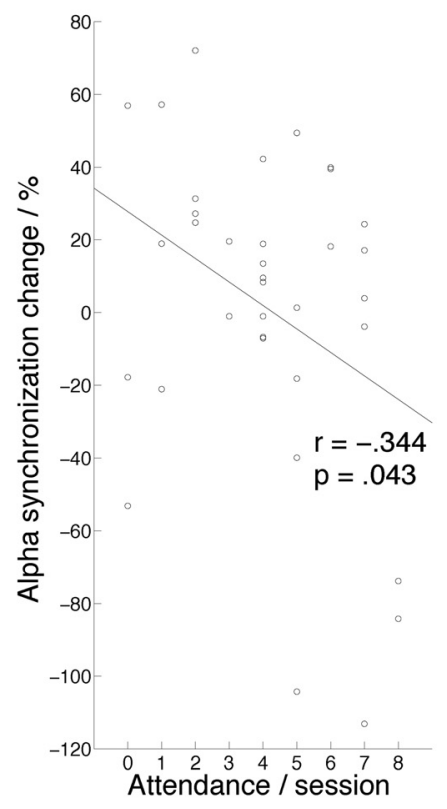

D

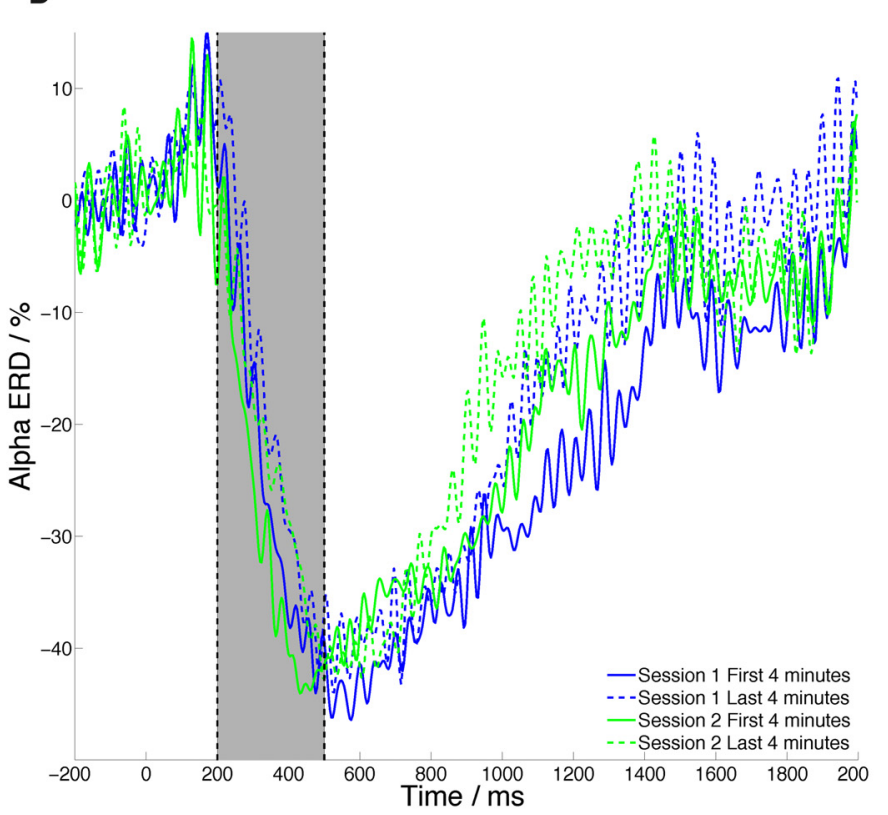

FIGURE 5 | Grand average event related desynchronization (ERD) topographic plot during PVT performance. (A) Before intervention. (B) After intervention. (C) ERD change across task and session is significantly correlated with MBT attendance. (D) Grand average ERD.

PVT bout (Figure 4). Using repeated-measures ANCOVA, we found a significant effect of session $\left(F_{(1,33)}=6.829, p=0.013\right.$, $\left.\eta_{\mathrm{p}}^{2}=0.171\right)$ and time $\left(F_{(1,33)}=9.049, p=0.005, \eta_{\mathrm{p}}^{2}=0.215\right)$ on this variable. The interaction between session and time was also significant $\left(F_{(1,33)}=7.667, p=0.009, \eta_{\mathrm{p}}^{2}=0.189\right)$, driven by smaller decreases in P3 amplitude over time in the post intervention session. ERP change values over time and session were not significantly correlated with attendance $(r=-0.259$, $p=0.133)$.

\section{ERD Analysis}

The same criteria for trial averaging were used for ERDs (Figure 5) and ERPs. There was no significant effect of session on alpha $\operatorname{ERD}\left(F_{(1,33)}=0.157, p=0.694, \eta_{\mathrm{p}}^{2}=0.005\right)$ and a trend effect of time $\left(F_{(1,33)}=3.681, p=0.064, \eta_{\mathrm{p}}^{2}=0.1\right)$. The session by time interaction was marginally significant $\left(F_{(1,33)}=3.472\right.$, $\left.p=0.71, \eta_{\mathrm{p}}^{2}=0.093\right)$.
ERD changes across time and session (i.e., the change in the time-on-task effect on ERDs) were significantly negatively correlated with number of the MBT sessions attended (Figure 5C; $r=-0.344, p=0.043$ ), demonstrating reduced alpha power synchronization with more sessions of MBT training attended.

\section{Subjective Data}

One participant did not provide self-report data in S2, and was excluded from the analysis. We found a significant effect of time $\left(F_{(1,33)}=5.679, p=0.023, \eta_{\mathrm{p}}^{2}=0.147\right)$ and a significant interaction between session and time $\left(F_{(1,33)}=5.034, p=0.032, \eta_{\mathrm{p}}^{2}=0.132\right)$ on subjective energy. When energy changes were correlated with attendance, we found that nurses who attended more sessions reported smaller decreases in subjective energy following PVT performance $(r=0.372, p=0.028)$. No significant changes were seen in subjective mood scales. 


\section{DISCUSSION}

In this intervention study, we investigated the effects of MBT on a widely used test of sustained attention: the Psychomotor Vigilance Test (PVT). In this experiment, we measured behavioral, subjective, and electrophysiological changes during performance of the 20-min PVT in a group of nurses before and after an 8-week MBT protocol. Attendance to the course was relatively low, and perhaps because of this, we did not find support for the hypotheses that sustained attention and its associated EEG markers would improve significantly in the whole group of trainees. However, when attendance was introduced as a covariate, our data show significant changes in PVT response speed and lapse counts, with better performance observed in nurses who attended more of the MBT. Similarly, we observed a significant session by time interaction in P3 amplitude, with high attendees showing a smaller time-on-task effect. Finally, nurses who attended more of the intervention reported lower subjective energy decreases as a result of performing the PVT. Overall, these data suggest that mindfulness training enhances the ability to sustain attention, with associated and measurable neurophysiological and subjective changes.

\section{MBT Has a Beneficial Effect on Sustained Attention}

Evidence of enhanced sustained attention has been found in those who already have meditation experience compared to novice meditators (Valentine and Sweet, 1999; Josefsson and Broberg, 2011). However, studies examining the effects of short-term mindfulness training on sustained attention have yielded mixed results. For example, studies using the attention network test (ANT) have shown both improvements and null effects in all three (alerting, orienting, executive) attentional networks in different experimental settings (Jha et al., 2007; Tang et al., 2007). In contrast, no differences were found on CPT performance by Halperin et al. (1991) or Anderson et al. (2007) in studies comparing MBT to a waitlist control or treatment as usual. Most recently, using the SART, Jha et al. (2015) found that MBT improved accuracy (measured by A') on this task in both a military and a civilian cohort.

The heterogeneity of study designs and outcome measures in the literature makes it challenging to draw conclusions from the currently available data. Furthermore, none of the studies above assessed sustained attention using the PVT, which is particularly useful for the assessment of operational readiness (Balkin et al., 2004). Importantly, the PVT is highly sensitive to changes to changes in alertness and vigilance due to factors such as sleep deprivation (Lim and Dinges, 2010), and shift work (Neri et al., 2002), and is suitable for measuring inter-individual differences in sustained attention across these states (Van Dongen and Dinges, 2005).

Analysis of the PVT data revealed a pattern of behavior over time whereby those with poor attendance in the MBT program tended to have relatively worse performance, while regular attendees showed a slight tendency to improve. Duration of sleep reported before each testing session did not differ significantly, suggesting that our results were not caused by differences in homeostatic sleep pressure. As the test-retest reliability of the PVT performed in a similar state is high (0.88; Dorrian et al., 2005), it is likely that the changes observed over time are due to enhancement or attenuation of the capacity to sustain attention. This improvement may be due to a number of different factors including increased awareness of mind wandering and other off-task behavior (Cheyne et al., 2009; Mrazek et al., 2013), an expanded resource pool of attentional resources to deploy towards on-task behavior, or better alertness due to improved sleep (Black et al., 2015).

We note that the overall comparison of response speed and lapses showed that performance was significantly worse in S2. This effect was driven largely by poor attendees. The data suggest that the high-stress environment in the workplace had adverse cognitive consequences on nurses' cognitive functioning (Shapiro et al., 2005; Yang et al., 2016; Burton et al., 2017), but that mindfulness training mitigated and even reversed the negative effects of the stress. Our results echo the findings of Leonard et al. (2013), who also found declines in attention over a similar span of time in a group of incarcerated youth, with protective effects conferred by MBT.

\section{MBT Reduces the Energetic Cost of Sustaining Attention}

In addition to the objective changes on the PVT, we observed that performing the PVT resulted in overall reductions in subjective feelings of energy (i.e., increases in sleepiness and fatigue), but that nurses with higher MBT attendance experienced a lower magnitude of decrease. Reductions in energy due to sustaining attention are commonly reported in the literature (Warm et al., 2008; Helton and Russell, 2011; Lim et al., 2012), and are thought to reflect resource expenditure due to task demands. Thus, the findings in the subjective scales support the theory that MBT induces either in greater availability or more efficient deployment of attentional resources during times of high resource demand (Thomson et al., 2015). This result may be of direct relevance to reducing the mental fatigue caused by performing nursing duties that is an indirect cause of nursing errors (Mayo and Duncan, 2004).

\section{MBT Enhances Alpha Power During Meditation}

We measured EEG power during a 4-min period of guided mindful breathing in order to assess spectral differences associated with MBT. We focused our analysis on the alpha and theta bands of the EEG spectrum, as these bands have shown the most robust changes following such training in the prior literature (Lomas et al., 2015). Consistent with this literature, we found significant differences across regions in the alpha band, with greater alpha increases associated with higher MBT attendance. This suggests that the intervention was effective in teaching participants who attended the program how to achieve a mindful state. No differences were found in the theta band.

Changes in alpha power are putatively driven by increases in internally directed attention (Klimesch et al., 2007), 
corresponding with relative elevation in activity in task-negative brain regions (Knyazev et al., 2011). Thus, our results suggest that participants were better able to focus attention internally and inhibit distraction/mind-wandering during meditation following MBT, but only if they had relatively high attendance to the program.

Theta power is an index of executive function, and it has been suggested that greater theta synchronization in meditators marks the increased capacity to control one's locus of attention (Cahn and Polich, 2006). However, in contrast with alpha, differences in theta power are not as consistently reported in experienced meditators, and indeed, we did not observe significant theta associations in the current work. This suggests that there may be factors (e.g., length of experience, type of meditative discipline) that moderate theta power differences in the mindful state after MBT.

\section{MBT Reduces P3 Attenuation Over Time During Task Performance}

We analyzed the P3 component of responses to PVT targets and found a significant effect of the intervention when attendance was included as a covariate. Although the direct correlation did not reach significance, $\mathrm{P} 3$ amplitude tended to be less attenuated with time-on-task when participants attended more of the MBT program. In undemanding tasks, the P3 is thought to be an index of resource allocation with greater peak amplitude signifying greater arousal and attention (Polich, 2007). As such, our data suggest that MBT changed either the ability or the willingness of participants to deploy resources consistently over the full duration of the PVT.

Previous studies of mindfulness practitioners have been consistent in finding modulation of the P3 component in comparison with controls (for a review, see Cahn and Polich, 2006). These changes typically fall into one of three categories.

First, several studies have reported reductions in P3 amplitude to salient but task-irrelevant stimuli in mindfulness practitioners, suggesting that superior attention in this group is due to an improvement in top-down inhibition. For example, Cahn and Polich (2009) studied experienced Vipassana meditators as they performed an auditory oddball task, and found P3a amplitude differences (as well as differences in $\mathrm{N} 1$ and $\mathrm{P} 2$ ) to rare distracter stimuli, but not to standard stimuli or oddballs. In a similar fashion, Moore et al. (2012) found that participants in the mindfulness-training arm of a randomized control trial (vs. a waitlisted group) had reduced P3 amplitude to incongruent stimuli on a color-word Stroop test.

Second, MBT can also enhance P3 amplitude to attended and relevant stimuli. Experienced Vipassana meditators show greater P3b amplitudes to targets on an auditory oddball task after meditation (Delgado-Pastor et al., 2013). Lakey et al. (2011) showed that a brief mindfulness induction boosted P3 amplitudes and improved accuracy in a brain-computer interface task. More recently, Smart et al. (2016) demonstrated such increases in a group of patients with subjective cognitive impairment (but not healthy controls) who performed a Go/No-Go task before and after MT.
Finally, there is evidence that MT can change the way in which limited attentional resources are deployed over time. In an elegant study of the attentional blink phenomenon, Slagter et al. (2007) showed that, following a 3-month meditation retreat, P3b amplitude was reduced for initial targets (reflecting lower attentional capture), thus reducing attentional-blink size, and improving detection of the second (blink) target. These data suggest a potential unifying mechanism for the changes described in the prior two paragraphs-meditation-trained participants might improve the ability both to upregulate resources devoted to relevant stimuli and downregulate resources devoted to distracting stimuli based on overall resource reallocation.

As the PVT only has a single stimulus type (in contrast to Go/No-Go or oddball tasks), we cannot make nuanced inferences about the functional significance of the P3 in this task. Nevertheless, our data are broadly compatible with the theory that $\mathrm{MBT}$ results in a more optimal allocation of resources to task-relevant behavior (Davies and Parasuraman, 1977). Additionally, our results suggest that these resources are more evenly deployed over time, mitigating the traditional declines in P3 amplitude that are seen with time-on-task (Koelega et al., 1992; Möckel et al., 2015; Staub et al., 2015).

\section{MBT Stabilizes Alpha ERD Over Time During Task Performance}

The final noteworthy result observed in this study was a significant reduction in the change in alpha-band ERD to target stimuli across the PVT from S1 to S2. Desynchronization in this band to targets reflects suppression of background noise and devotion of processing resources to the relevant stimulus (Pfurtscheller and Lopes da Silva, 1999). As with P3 amplitude, we observed a significant effect of time on ERD, suggesting that participants found it increasingly difficult to effectively allocate attentional resources to the task. Following MBT, those with good attendance were less vulnerable to this effect over time, possibly because of an improved ability to suppress background neural noise.

\section{Limitations}

The primary limitation of the current study is the lack of a control group, or randomization to condition, the gold standard of any intervention study being a double-blind randomized controlled trial. Due to logistical constraints, we were not able to conduct this form of trial in this study. As our design uses comparisons of pre- and post-intervention outcomes, we cannot make strong causal claims about whether MBT per se led to our observed changes. Nevertheless, one merit of the current design is that it is a good reflection of the likely results of a MBT course being offered in the real world.

Second, it may be possible that differences in motivation might account for the results. Individuals participating in the program who are more highly motivated would likely attend more of the training may put in more effort while performing the PVT whilst participants less invested in the program may attend less or none of the training and put 
minimal effort into PVT performance. To address this point, we compared motivation across sessions using one of the items administered on the pre-task questionnaire ("How motivated are you"). Using a repeated measures ANCOVA with attendance as a covariate, we found no significant difference on this item $\left(F_{(1,34)}=3.356, p=0.76, \eta^{2}=0.09\right)$, indicating that motivation was unlikely to account for the other observed changes.

Third, attendance to our program was relatively poor, limiting our ability to study the effect of a full MBT course on changes in cognition. Other investigators have also reported that it can be challenging to get participant compliance with mindfulness training in workplace settings (Van Dongen et al., 2016). Nevertheless, as a consequence of this spread in attendance, we were able to provide evidence that sustained attention might get worse over time in a stressful working environment, and that it may be important for those undergoing mindfulness training to attend a majority of the training sessions in order to reap its benefits on attention.

\section{CONCLUSION}

In summary, our data support the hypothesis that attending MBT is an effective means of mitigating or reversing the negative effects of work stress on sustained attention. However, we caution that less weight should be placed on the evidence presented here in comparison with other studies using stronger designs, particularly randomized controlled trials.

\section{REFERENCES}

Anderson, N. D., Lau, M. A., Segal, Z. V., and Bishop, S. R. (2007). Mindfulnessbased stress reduction and attentional control. Clin. Psychol. Psychother. 14, 449-463. doi: 10.1002/cpp.544

Balkin, T. J., Bliese, P. D., Belenky, G., Sing, H., Thorne, D. R., Thomas, M., et al. (2004). Comparative utility of instruments for monitoring sleepinessrelated performance decrements in the operational environment. J. Sleep Res. 13, 219-227. doi: 10.1111/j.1365-2869.2004.00407.x

Barger, L. K., Ayas, N. T., Cade, B. E., Cronin, J. W., Rosner, B., Speizer, F. E., et al. (2006). Impact of extended-duration shifts on medical errors, adverse events and attentional failures. PLoS Med. 3:e487. doi: 10.1371/journal.pmed. 0030487

Basner, M., and Dinges, D. F. (2011). Maximizing sensitivity of the psychomotor vigilance test (PVT) to sleep loss. Sleep 34, 581-591. doi: 10.1093/sleep/34.5.581

Benner, P., Sheets, V., Uris, P., Malloch, K., Schwed, K., and Jamison, D. (2002). Individual, practice and system causes of errors in nursing: a taxonomy. J. Nurs. Adm. 32, 509-523. doi: 10.1097/00005110-200210000-00006

Black, D. S., O’Reilly, G. A., Olmstead, R., Breen, E. C., and Irwin, M. R. (2015). Mindfulness meditation and improvement in sleep quality and daytime impairment among older adults with sleep disturbances: a randomized clinical trial. JAMA Intern. Med. 175, 494-501. doi: 10.1001/jamainternmed. 2014.8081

Bonnet, M. H., Gomez, S., Wirth, O., and Arand, D. L. (1995). The use of caffeine versus prophylactic Naps in sustained performance. Sleep 18, 97-104. doi: 10.1093/sleep/18.2.97

Brainard, D. H. (1997). The psychophysics toolbox. Spat. Vis. 10, 433-436. doi: $10.1163 / 156856897 \times 00357$

Burton, A., Burgess, C., Dean, S., Koutsopoulou, G. Z., and Hugh-Jones, S. (2017). How effective are mindfulness-based interventions for reducing stress among healthcare professionals? A systematic review and meta-analysis. Stress Health 33, 3-13. doi: 10.1002/smi.2673
Attention is a prerequisite for higher cognitive operations, and failures of sustained attention or vigilance can lead to serious errors, particularly in medical practice (Barger et al., 2006; Scott et al., 2006). Here, we show a tripartite benefit of MBT on this domain, with a protective effect on behavior, electrophysiology, and subjective energy expenditure. MBT is beginning to show promise as a low-cost, easily disseminable means of combating failures of sustained attention, and further study is warranted to reveal the active ingredients of this intervention and the best ways in which it may be delivered.

\section{AUTHOR CONTRIBUTIONS}

The study design was concieved by JL, KD and MWLC. Stimuli was prepared by KFW and KD. Data was collected by JT and KFW. Data analysis was performed by KFW. JL, KFW and MWLC contributed to writing of the manuscript.

\section{ACKNOWLEDGMENTS}

This study was funded by a gift from the Far East Organization to MWLC. We acknowledge the help of Chong Shin Wee and Stacey Lee Henderson in data collection, and the support of Dr. Tan Siok Bee, Deputy Director of Nursing, as well as Ms. Fazila Aloweni and Chua Tse Lert from the Division of Nursing Research who assisted with advertising, recruitment and data collection.

Cahn, B. R., and Polich, J. (2006). Meditation states and traits: EEG, ERP, and neuroimaging studies. Psychol. Bull. 132, 180-211. doi: 10.1037/0033-2909.132. 2.180

Cahn, B. R., and Polich, J. (2009). Meditation (Vipassana) and the P3a eventrelated brain potential. Int. J. Psychophysiol. 72, 51-60. doi: 10.1016/j.ijpsycho. 2008.03.013

Cheyne, J. A., Solman, G. J. F., Carriere, J. S. A., and Smilek, D. (2009). Anatomy of an eror: bidirectional state model of task engagement/disengagement and attention-related errors. Cognition 111, 98-113. doi: 10.1016/j.cognition.2008. 12.009

Chiesa, A., Calati, R., and Serretti, A. (2011). Does mindfulness training improve cognitive abilities? A systematic review of neuropsychological findings. Clin. Psychol. Rev. 31, 449-464. doi: 10.1016/j.cpr.2010.11.003

Chiesa, A., and Serretti, A. (2010). A systematic review of neurobiological and clinical features of mindfulness meditations. Psychol. Med. 40, 1239-1252. doi: $10.1017 /$ S0033291709991747

Davies, D. R., and Parasuraman, R. (1977). "Cortical evoked potentials and vigilance: a decision theory analysis," in Vigilance, ed. R. R. Mackie (Boston, MA: Springer), 285-306.

Delgado-Pastor, L. C., Perakakis, P., Subramanya, P., Telles, S., and Vila, J. (2013). Mindfulness (Vipassana) meditation: effects on P3b event-related potential and heart rate variability. Int. J. Psychophysiol. 90, 207-214. doi: 10.1016/j.ijpsycho. 2013.07.006

Delorme, A., and Makeig, S. (2004). EEGLAB: an open source toolbox for analysis of single-trial EEG dynamics including independent component analysis. J. Neurosci. Methods 134, 9-21. doi: 10.1016/j.jneumeth.2003. 10.009

Dinges, D. F. (1995). An overview of sleepiness and accidents. J. Sleep Res. 4, 4-14. doi: 10.1111/j.1365-2869.1995.tb00220.x

Dinges, D. F. (2016). Psychomotor vigilance self test on the international space station (reaction self test) - 08.10.16. Available online at: http://www.nasa. gov/mission_pages/station/research/experiments/982.html 
Dinges, D. F., and Kribbs, N. B. (1991). "Performing while sleepy: effects of experimentally-inducing sleepiness," in Sleep, Sleepiness and Performance (Vol. XIV), ed. T. H. Monk (Oxford, England: John Wiley and Sons), 96-128.

Dinges, D. F., and Powell, J. W. (1985). Microcomputer analyses of performance on a portable, simple visual RT task during sustained operations. Behav. Res. Methods Instrum. Comput. 17, 652-655. doi: 10.3758/bf03200977

Dorrian, J., Rogers, N. L., and Dinges, D. F. (2005). "Psychomotor vigilance performance: neurocognitive assay sensitive to sleep loss," in Sleep Deprivation, ed. C. A. Kushida (New York, NY: Marcel Dekker), 39-70.

Halperin, J. M., Shrama, V., Greenblatt, E., and Schwartz, S. T. (1991). Assessment of the continuous performance test: reliability and validity in a nonreferred sample. Psychol. Assess. 3, 603-608. doi: 10.1037//1040-3590.3.4.603

Härkänen, M., Turunen, H., Saano, S., and Vehviläinen-Julkunen, K. (2013). Medication errors: what hospital reports reveal about staff views. Nurs. Manag. (Harrow) 19, 32-37. doi: 10.7748/nm2013.03.19.10.32.e1010

Helton, W. S., and Russell, P. N. (2011). Working memory load and the vigilance decrement. Exp. Brain Res. 212, 429-437. doi: 10.1007/s00221-011-2749-1

Jankowski, T., and Holas, P. (2014). Metacognitive model of mindfulness. Conscious. Cogn. 28, 64-80. doi: 10.1016/j.concog.2014.06.005

Jha, A. P., Krompinger, J., and Baime, M. J. (2007). Mindfulness training modifies subsystems of attention. Cogn. Affect. Behav. Neurosci. 7, 109-119. doi: 10.3758/cabn.7.2.109

Jha, A. P., Morrison, A. B., Dainer-Best, J., Parker, S., Rostrup, N., and Stanley, E. A. (2015). Minds "at attention": mindfulness training curbs attentional lapses in military cohorts. PLoS One 10:e116889. doi: 10.1371/journal.pone.0116889

Josefsson, T., and Broberg, A. (2011). Meditators and non-meditators on sustained and executive attentional performance. Ment. Health Relig. Cult. 14, 291-309. doi: 10.1080/13674670903578621

Kabat-Zinn, J., Lipworth, L., and Burney, R. (1985). The clinical use of mindfulness meditation for the self-regulation of chronic pain. J. Behav. Med. 8, 163-190. doi: $10.1007 /$ bf00845519

Kaul, P., Passafiume, J., Sargent, R. C., and O’Hara, B. F. (2010). Meditation acutely improves psychomotor vigilance and may decrease sleep need. Behav. Brain Funct. 6:47. doi: 10.1186/1744-9081-6-47

Klimesch, W. (1999). EEG alpha and theta oscillations reflect cognitive and memory performance: a review and analysis. Brain Res. Rev. 29, 169-195. doi: 10.1016/s0165-0173(98)00056-3

Klimesch, W., Sauseng, P., and Hanslmayr, S. (2007). EEG alpha oscillations: the inhibition-timing hypothesis. Brain Res. Rev. 53, 63-88. doi: 10.1016/j. brainresrev.2006.06.003

Knyazev, G. G., Slobodskoj-Plusnin, J. Y., Bocharov, A. V., and Pylkova, L. V. (2011). The default mode network and EEG alpha oscillations: an independent component analysis. Brain Res. 1402, 67-79. doi: 10.1016/j.brainres.2011. 05.052

Koelega, H. S., Verbaten, M. N., van Leeuwen, T. H., Kenemans, J. L., Kemner, C., and Sjouw, W. (1992). Time effects on event-related brain potentials and vigilance performance. Biol. Psychol. 34, 59-86. doi: 10.1016/03010511(92)90024-o

Lakey, C. E., Berry, D. R., and Sellers, E. W. (2011). Manipulating attention via mindfulness induction improves P300-based brain-computer interface performance. J. Neural Eng. 8:025019. doi: 10.1088/1741-2560/8/2/025019

Leonard, N. R., Jha, A. P., Casarjian, B., Goolsarran, M., Garcia, C., Cleland, C. M., et al. (2013). Mindfulness training improves attentional task performance in incarcerated youth: a group randomized controlled intervention trial. Front. Psychol. 4:792. doi: 10.3389/fpsyg.2013.00792

Lim, J., Bogossian, F., and Ahern, K. (2010). Stress and coping in Singaporean nurses: a literature review. Nurs. Health Sci. 12, 251-258. doi: 10.1111/j.14422018.2010.00514.x

Lim, J., and Dinges, D. F. (2008). Sleep deprivation and vigilant attention. Ann. N Y Acad. Sci. 1129, 305-322. doi: 10.1196/annals.1417.002

Lim, J., and Dinges, D. F. (2010). A meta-analysis of the impact of short-term sleep deprivation on cognitive variables. Psychol. Bull. 136, 375-389. doi: $10.1037 / \mathrm{a} 0018883$

Lim, J., Ebstein, R., Tse, C. Y., Monakhov, M., Lai, P. S., Dinges, D. F., et al. (2012). Dopaminergic polymorphisms associated with time-on-task declines and fatigue in the psychomotor vigilance test. PLoS One 7:e33767. doi: 10.1371/journal.pone.0033767
Lomas, T., Ivtzan, I., and Fu, C. H. Y. (2015). A systemic review of the neurophysiology of mindfulness on EEG oscillations. Neurosci. Biobehav. Rev. 57, 401-410. doi: 10.1016/j.neubiorev.2015.09.018

MacCoon, D. G., MacLean, K. A., Davidson, R. J., Saron, C. D., and Lutz, A. (2014). No sustained attention differences in a longitudinal randomized trial comparing mindfulness based stress reduction versus active control. PLoS One 9:e97551. doi: 10.1371/journal.pone.0097551

Mayo, A. M., and Duncan, D. (2004). Nurse perceptions of medication errors: what we need to know for patient safety. J. Nurs. Care Qual. 19, 209-217. doi: 10.1097/00001786-200407000-00007

Möckel, T., Beste, C., and Wascher, E. (2015). The effects of time on task in response selection-an ERP study of mental fatigue. Sci. Rep. 5:10113. doi: 10.1038/srep10113

Moore, A., Gruber, T., Derose, J., and Malinowski, P. (2012). Regular, brief mindfulness meditation practice improves electrophysiological markers of attentional control. Front. Hum. Neurosci. 6:18. doi: 10.3389/fnhum.2012. 00018

Mrazek, M. D., Franklin, M. S., Phillips, D. T., Baird, B., and Schooler, J. W. (2013). Mindfulness training improves working memory capacity and GRE performance while reducing mind wandering. Psychol. Sci. 24, 776-781. doi: 10.1177/0956797612459659

Neri, D. F., Oyung, R. L., Colletti, L. M., Mallis, M. M., Tam, P. Y., and Dinges, D. F. (2002). Controlled breaks as a fatigue countermeasure on the flight deck. Aviat. Space Environ. Med. 73, 654-664.

Parasuraman, R., and Jiang, Y. (2012). Individual differences in cognition, affect, and performance: behavioral, neuroimaging, and molecular genetic approaches. Neuroimage 59, 70-82. doi: 10.1016/j.neuroimage.2011.04.040

Pfurtscheller, G., and Lopes da Silva, F. H. (1999). Event-related EEG/MEG synchronization and desynchronization: basic principles. Clin. Neurophysiol. 110, 1842-1857. doi: 10.1016/s1388-2457(99)00141-8

Polich, J. (2007). Updating P300: an integrative theory of P3a and P3b. Clin. Neurophysiol. 118, 2128-2148. doi: 10.1016/j.clinph.2007.04.019

Polich, J., and Kok, A. (1995). Cognitive and biological determinants of P300: an integrative review. Biol. Psychol. 41, 103-146. doi: 10.1016/03010511(95)05130-9

Scott, L. D., Rogers, A. E., Huang, W. T., and Zhang, Y. (2006). Effects of critical care nurses' work hours on vigilance and patients' safety. Am. J. Crit. Care 15, 30-37.

Shapiro, S. L., Astin, J. A., Bishop, S. R., and Cordova, M. (2005). Mindfulness-based stress reduction for health care professionals: results from a randomized trial. Int. J. Stress Manag. 12, 164-176. doi: 10.1037/1072-5245. 12.2.164

Slagter, H. A., Lutz, A., Greischar, L. L., Francis, A. D., Nieuwenhuis, S., Davis, J. M., et al. (2007). Mental training affects distributions of limited brain resources. PLoS Biol. 5:e138. doi: 10.1371/journal.pbio.0050138

Smart, C. M., Segalowitz, S. J., Mulligan, B. P., Koudys, J., and Gawryluk, J. R. (2016). Mindfulness training for older adults with subjective cognitive decline: results from a pilot randomized controlled trial. J. Alzheimers Dis. 52, 757-774. doi: $10.3233 /$ jad-150992

Staub, B., Doignon-Camus, N., Marques-Carneiro, J. E., Bacon, E., and Bonnefond, A. (2015). Age-related differences in the use of automatic and controlled processes in a situation of sustained attention. Neuropsychologia 75, 607-616. doi: 10.1016/j.neuropsychologia.2015.07.021

Tang, Y. Y., Ma, Y., Wang, J., Fan, Y., Feng, S., Lu, Q., et al. (2007). Short-term meditation training improves attention and self-regulation. Proc. Natl. Acad. Sci. U S A 104, 17152-17156. doi: 10.1073/pnas.0707678104

Thomson, D. R., Besner, D., and Smilek, D. (2015). A resource-control account of sustained attention: evidence from mind-wandering and vigilance paradigms. Perspect. Psychol. Sci. 10, 82-96. doi: 10.1177/1745691614556681

Tucker, P., Folkard, S., and Macdonald, I. (2003). Rest breaks and accident risk. Lancet 361:680. doi: 10.1016/s0140-6736(03)12566-4

Valentine, E. R., and Sweet, P. L. G. (1999). Meditation and attention: a comparison of the effects of concentrative and mindfulness meditation on sustained attention. Ment. Health Relig. Cult. 2, 59-70. doi: 10.1080/13674679908406332

Van Dongen, H. P., and Dinges, D. F. (2005). Sleep, circadian rhythms and psychomotor vigilance. Clin. Sports Med. 24, 237-249. doi: 10.1016/j.csm.2004. 12.007 
Van Dongen, J. M., Van Berkel, J., Boot, C. R. L., Bosmans, J., Proper, K., Bonger, P. M., et al. (2016). Long-term cost-effectiveness and returnon-investment of a mindfulness-based worksite intervention: results of a randomized controlled trial. J. Occup. Environ. Med. 58, 550-560. doi: 10.1097/jom.0000000000000736

Warm, J. S., Parasuraman, R., and Matthews, G. (2008). Vigilance requires hard mental work and is stressful. Hum. Factors 50, 433-441. doi: 10.1518/001872008X312152

Welch, P. (1967). The use of fast Fourier transform for the estimation of power spectra: a method based on time averaging over short, modified periodograms. IEEE Trans. Audio Electroacoust. 15, 70-73. doi: 10.1109/tau.1967. 1161901

Yang, S., Meredith, P., and Khan, A. (2016). Is mindfulness associated with stress and burnout among mental health professionals in Singapore? Psychol. Health Med. 22, 673-679. doi: 10.1080/13548506.2016.1220595
Yordanova, J., Kolev, V., and Polich, J. (2001). P300 and alpha event-related desynchronization (ERD). Psychophysiology 38, 143-152. doi: 10.1111/14698986.3810143

Conflict of Interest Statement: The authors declare that the research was conducted in the absence of any commercial or financial relationships that could be construed as a potential conflict of interest.

Copyright (C) 2018 Wong, Teng, Chee, Doshi and Lim. This is an open-access article distributed under the terms of the Creative Commons Attribution License (CC BY). The use, distribution or reproduction in other forums is permitted, provided the original author(s) and the copyright owner are credited and that the original publication in this journal is cited, in accordance with accepted academic practice. No use, distribution or reproduction is permitted which does not comply with these terms. 